\title{
Deep Dive with Contagion: COVID-19
}

COVID-19, short for "coronavirus disease 2019," is the official name given by the World Health Organization to the disease caused by this newly identified coronavirus. It is caused by a member of the coronavirus family that has never been encountered before. Like other coronaviruses, it has transferred to humans from animals. It has spread so rapidly and to so many countries that the World Health Organization has declared it a pandemic. COVID-19 is caused by a coronavirus called SARS-CoV-2. Coronaviruses are a large family of viruses that are common in people and many different species of animals, including camels, cattle, cats, and bats. Rarely, animal coronaviruses can infect people and then spread between people. The virus that causes COVID-19 is thought to spread mainly from person to person, mainly through respiratory droplets produced when an infected person coughs or sneezes. These droplets can land in the mouths or noses of people who are nearby or possibly be

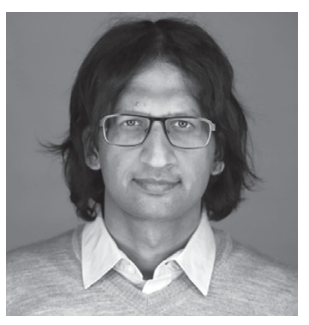
inhaled into the lungs. Spread is more likely when people are in close contact with one another (within about 6 feet). The incubation period for COVID-19, which is the time between exposure to the virus (becoming infected) and symptom onset, is on average 5-6 days, however, can be up to 14 days. During this period, also known as the "pre-symptomatic" period, some infected persons can be contagious. Therefore, transmission from a pre-symptomatic case can occur before symptoms onset. The primary symptoms in these patients include fever, dry cough, tiredness, pain in the pharynx (pharyngalgia) and difficult or labored breathing (dyspnea). Some patients may also have a runny nose, sore throat, nasal congestion and aches and pains or diarrhea. Some people report losing their sense of taste and/or smell. Coronavirus infections are diagnosed by a healthcare provider based on symptoms and are confirmed through laboratory tests. About $80 \%$ of people who get COVID-19 experience a mild case - about as serious as a regular cold - and recover without needing any special treatment. The median time from onset to clinical recovery for mild cases is approximately 2 weeks and is 3-6 weeks for patients with severe or critical disease. Based on the currently available information and clinical expertise, older adults and people of any age who have serious underlying medical conditions, might be at higher risk for severe illness from COVID-19. Spreading the infection can be prevented by social distancing, washing hands regularly or alcohol-based hand rub, covering nose and mouth with a disposable tissue or flexed elbow when cough or sneeze and self-isolate from others in the household if feeling unwell. Nevertheless, to stop the contagion spread of coronavirus pandemic, "Prevention is always better than cure."

Rajiv Saini

Editor-in-Chief International Journal of Experimental Dental Science 